\title{
MMP7 interacts with ARF in nucleus to potentiate tumor microenvironments for prostate cancer progression in vivo
}

\author{
Yingqiu Xie ${ }^{1}$, Wenfu Lu ${ }^{2}$, Shenji Liu ${ }^{2}$, Qing Yang${ }^{2}$, J. Shawn Goodwin², Sandeep \\ Anantha Sathyanarayana ${ }^{3}$, Siddharth Pratap ${ }^{4}$, Zhenbang Chen ${ }^{2}$ \\ ${ }^{1}$ Department of Biology, School of Science and Technology, Nazarbayev University, Astana, 010000, Republic of Kazakhstan \\ ${ }^{2}$ Department of Biochemistry and Cancer Biology, Meharry Medical College, Nashville, TN, 37208, USA \\ ${ }^{3}$ Department of Surgery, Meharry Medical College, Nashville, TN, 38208, USA \\ ${ }^{4}$ School of Graduate Studies and Research, Meharry Medical College, Nashville, TN, 37208, USA \\ Correspondence to: Yingqiu Xie, email: yingqiu.xie@nu.edu.kz \\ Zhenbang Chen, email: zchen@mmc.edu
}

Keywords: $M M P 7, A R F$, tumor microenvironments, prostate cancer

Received: January 19, 2016 Accepted: June 12, $2016 \quad$ Published: June 23, 2016

\section{ABSTRACT}

ARF couples with TP53 in a canonical signaling pathway to activate cellular senescence for tumor suppressive function under oncogenic insults. However, the mechanisms on aberrant elevation of ARF in cancers are still poorly understood. We previously showed that ARF ( $14^{A R F}$ in human and p19 Arf in mouse) elevation correlates with PTEN loss and stabilizes SLUG to reduce cell adhesion in prostate cancer ( $P C a)$. Here we report that ARF is essential for MMP7 expression, E-Cadherin decrease and the anchorage loss to the extracellular matrix (ECM) in PCa in vitro and in vivo. We found that Mmp7 is aberrantly elevated in cytosol and nucleus of malignant prostate tumors of Pten/Trp53 mutant mice. Interestingly, p19Arf deficiency strikingly decreases Mmp7 levels but increases E-Cadherin in Pten/ Trp53/p19Arf mice. ARF knockdown markedly reduces MMP7 in human PCa cells. Conversely, tetracycline-inducible expression of ARF increases MMP7 with a decrease of E-Cadherin in PCa cells. Importantly, MMP7 physically binds ARF to show the co-localization in nucleus. Co-expression of MMP7 and ARF promotes cell migration, and MMP7 knockdown decreases wound healing in PCa cells. Furthermore, MMP7 elevation correlates with ARF expression in advanced human PCa. Our findings reveal for the first time that the crosstalk between ARF and MMP7 in nucleus contributes to ECM network in tumor microenvironments in vivo, implicating a novel therapeutic target for advanced PCa treatment.

\section{INTRODUCTION}

Prostate cancer $(\mathrm{PCa})$ is the second leading cause of cancer-related deaths among men in Western countries and the emerging threat to men in Asian countries $[1,2]$. Aberrations of multiple oncogenic pathways contribute to the initiation and progression of PCa [3-6]. Mutation and deletion of phosphatase and tensin homolog deleted on chromosome 10 (PTEN) are frequently found in human cancers, including PCa $[7,8]$. Pten/Trp53 mutant mice provide us a unique and powerful tool to elucidate potential oncogenic factors of PTEN network. However, complete pictures on the mechanisms leading to the striking features of malignancy are still poorly understood. ARF ( $\mathrm{p} 14^{\mathrm{ARF}}$ in human and p19 $9^{\text {Arf }}$ in mouse) elevation is found in PTENdeficient human $\mathrm{PCa}$ [9] and various cancer cell lines [10-12]. ARF is originally identified as an alternative transcript of ARF-INK4a locus on human chromosome 9q21 (chromosome 4 in mouse) [13]. Induction of the canonical ARF pathway halts cancer progression through coupling with TP53 to induce cellular senescence and inhibiting ribosomal RNA transcription and processing, response to DNA damage and autophagy initiation $[14,15]$. Nevertheless, ARF elevation is associated with the triggering of oncogenic pathways, which in turn results in additional alterations of molecular cascades for cancer progression. 
We previously demonstrated that ARF stabilizes SLUG to promote epithelial-mesenchymal transition (EMT) in PCa through degradation of cell adhesion [16]. The ECM, a key factor in cell adhesion and migration, is mainly degraded by matrix metalloproteinases (MMPs) [17]. MMP7, one of the secreted proteolytic enzymes, is associated with invasion and metastasis of cancers including PCa [18-20]. The mechanisms on oncogenic contributions of MMP7 to PCa progression in PTEN-null context still remain unclear. Given that Pten/Trp53 mice produce aggressive $\mathrm{PCa}$ through senescence evasion and p19 Arf elevation, we took advantage of this mouse model and bioinformatics approaches to investigate the noncanonical ARF signaling in cancers. Our results revealed a genetic landscape mediated by $\mathrm{p} 19^{\text {Arf }}$ in prostate tumors in vivo and further identified a novel ARF-MMP7 pathway in tumor microenvironments.

\section{RESULTS}

\section{Mmp7 expression is p19 ${ }^{\text {Arf }}$-dependent oncogenic signaling in Pten/Trp53 mouse model}

Pten/Trp53 mutant mice develop aggressive PCa with $\mathrm{p} 19^{\text {Arf }}$ upregulation and loss of epithelial adhesion [16]. In order to gain deep insights into the impact of $\mathrm{p} 19^{\text {Arf }}$ loss on oncogenic pathways in $\mathrm{PCa}$ in vivo, we applied bioinformatics approaches to investigate the changes of key factors on tumor microenvironments between Pten/ Trp53 and Pten/Trp53/p19Arf mutant mice. We performed the transcriptome profiling and histopathology analysis of prostate tumors in Pten/Trp53 and Pten/Trp 53/p19 Arf mice at 6 months of age ( 3 mice/group). Our data revealed that p19 ${ }^{\text {Arf }}$ loss (with one allele deletion of p19 ${ }^{\text {Arf }}$ ) resulted in both downregulation and upregulation of genes (Figure 1A), suggesting that $\mathrm{p} 19^{\text {Arf }}$ has biological functions on rRNA formation and protein synthesis, in addition to being a tumor suppressor. p19 Arf upregulates significantly a subset of oncogenes such as Mmp7, Mmp15 and Muc20, a MET signaling regulator (Supplementary Tables S1 and S2), at least in the context of Pten/Trp53 loss. Given that $M M P$ genes are primarily involved in ECM and cell adhesion, our results indicate that ARF may activate tumor microenvironments through MMP.

Since Mmp7 was dramatically decreased by p19 Arf loss in vivo, we decided to investigate the crosstalk between ARF and MMP7 in human PCa cells and to further explore whether ARF increases ECM regulator proteins for PCa progression. Immunohistochemistry (IHC) analysis revealed that levels of Mmp7, Snail and Vimentin were strikingly elevated in Pten/Trp53 tumors, but were dramatically decreased in Pten/Trp53/p19 Arf tumors upon p19 Arf deficiency (Figure 1B and Supplementary Figure S1). In agreement with the results, p19 ${ }^{\text {Arf }}$ deficiency increased E-Cadherin and decreased Ki67 in Pten/Trp53/p19Arf tumors (Figure 1B). Interestingly, Mmp7 was localized in nucleus of malignant cells in Pten/Trp53 tumors (Figure 1C), in addition to its localizations at membrane and cytoplasm. Our results support that nuclear MMPs are positively associated with aggressive features of tumors [21], and indicate that a concomitant elevation of MMP7 and ARF in nucleus may be critical for prostate tumorigenesis.

\section{Nuclear MMP7 is decreased by ARF knockdown in human prostate cancer cells}

Our analysis reveals that MMP7 protein contains the PKWXXKV sequence that is partially conserved with the nuclear localization signal (NLS) in MMP3 [18, 22, 23] (Figure 2A), suggesting an alternate mechanism on its shuttling between membrane/cytoplasm to nucleus. Since p19 ${ }^{\text {Arf }}$ loss decreases Mmp7 in mouse, we reasoned that nuclear MMP7 requires ARF and could be downregulated upon ARF loss in human PCa cells. To test the hypothesis and explore the interactions of MMP7 and ARF, we first examined protein levels of MMP7 and ARF in human PCa and normal cell lines. We found that MMP7 and ARF were highly expressed in PC3 and DU145 PCa cells, while their levels were very low in 22Rv1, LNCaP, C4-2B PCa cells and normal prostate cells. Interestingly, along with the elevation of MMP7 and ARF, levels of Vimentin were increased with a decrease of E-Cadherin in PC3 and DU145 cells (Figure 2B). We chose PC3 cells to knockdown p14 ${ }^{\mathrm{ARF}}$ by small hairpin RNA interference (shRNA). As confirmed with Western blot, p14 ${ }^{\mathrm{ARF}}$ knockdown resulted in a striking reduction of MMP7 as compared to the control (Figure 2C). The fractionation analysis revealed that the zymogen proform of MMP7 (pro-MMP7), not the active-MMP7, was decreased by $\mathrm{p} 14^{\mathrm{ARF}}$ knockdown. In addition, E-Cadherin was increased upon p14 ${ }^{\mathrm{ARF}}$ knockdown (Figure 2C), indicating that ARF-MMP7 network determines EMT phenotype in PCa. MMP7 in nucleus consists of the proMMP7 only (right panel, Figure 2C), suggesting that proMMP7 can shuttle between cytosol and nucleus without cleavage. We then performed immunofluorescence (IF) staining to detect the co-localization of MMP7 and ARF in PC3 and DU145 cells. In agreement with literature, MMP7 protein was localized predominantly at the cytosol and membrane of PC3 cells (Figure 2D). Surprisingly, MMP7 was also aberrantly localized in nucleus and co-localized with ARF in both PC3 and DU145 cells as confirmed with different antibodies (Supplementary Figure S2). p14 ${ }^{\mathrm{ARF}}$ knockdown abrogated MMP7 in nucleus of PC3 cells at both mRNA and protein levels (Figure 2D, Supplementary Figures S3 and S4). Our results indicate that MMP7 may promote EMT-like signaling through crosstalk with ARF.

\section{MMP7 is increased with E-Cadherin decrease upon ARF induction in prostate cancer cells}

We asked ourselves whether ARF upregulation would lead to an increase of MMP7, an opposing effect of p14 ${ }^{\mathrm{ARF}}$ knockdown on the signaling events in PCa cells. We sought to increase the endogenous levels of ARF in 22Rv1 cells 
by addition of IGF1. As a result, a concomitant elevation of ARF and MMP7 was indeed observed upon IGF stimulus as compared to the control (Figure 3A). We applied a TetOn inducible expression system to establish a doxycyclineinducible $\mathrm{p} 14^{\mathrm{ARF}}$ overexpression cell line from $\mathrm{C4}-2 \mathrm{~B}$ cells (Figure $3 \mathrm{~B}$ and $3 \mathrm{C}$ ). In this system, p14 ${ }^{\mathrm{ARF}}$ expression driven by a CMV promoter is repressed by Tet receptor but can be activated by addition of doxycycline, as it prevents Tet receptor from binding to the promoter. $\mathrm{p} 14^{\mathrm{ARF}}$ gene is conjugated with luciferase, so p14 ${ }^{\mathrm{ARF}}$ expression is indicated by luciferase activity in a dose-dependent manner upon addition of doxycycline. Importantly, p14 ${ }^{\mathrm{ARF}}$ induction resulted in a striking decrease of E-Cadherin and EMT phenotype (Figure 3C). Withdrawal of doxycycline reversed the EMT feature (Figure 3D). Similarly, p14 ${ }^{\mathrm{ARF}}$ induction increased MMP7 and SLUG with a decrease of E-Cadherin in LNCaP cells $(1.5 \mu \mathrm{g} / \mathrm{ml}$ doxycycline, 2 days) (Figure 3E, and panels below for the quantification). Furthermore, p14 ${ }^{\mathrm{ARF}}$ induction significantly promoted cell migration in LNCaP cells (Figure 3F). Together, our results suggest MMP7 contributes to cell migration and EMT, at least in part through ARF.
A

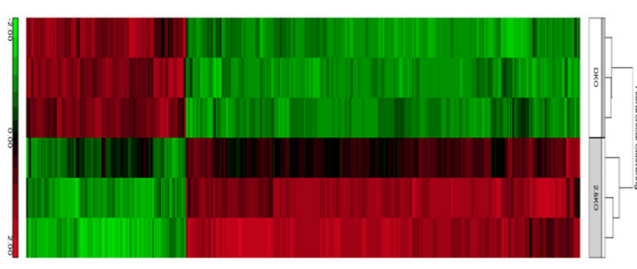

B
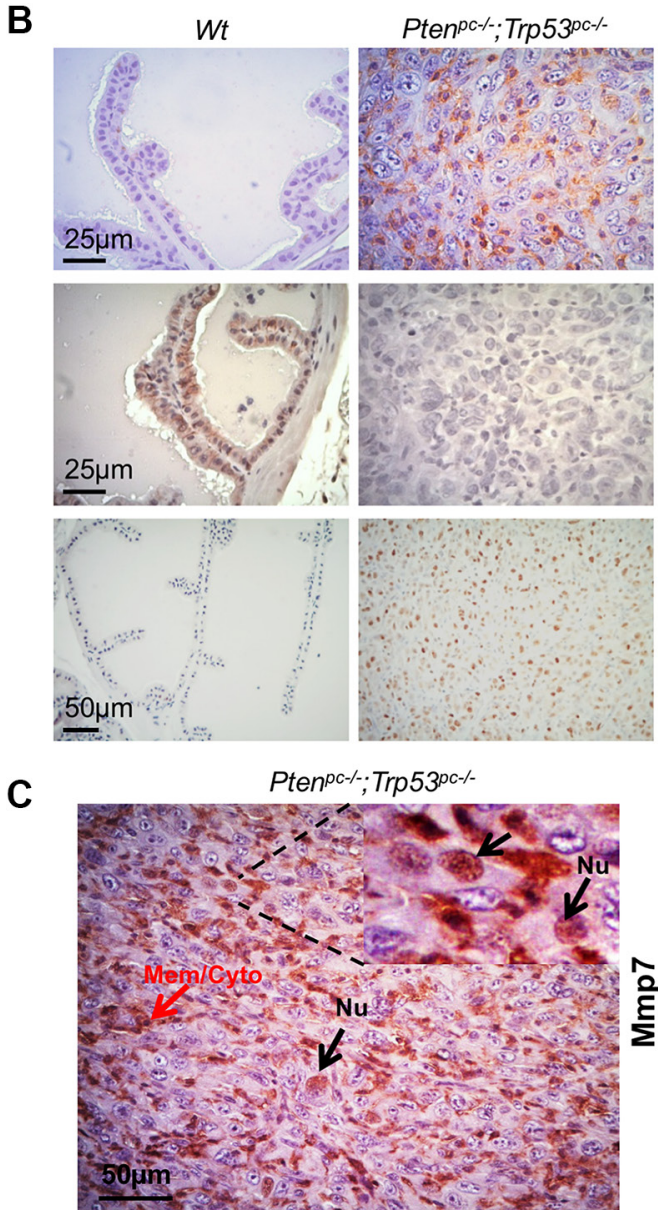

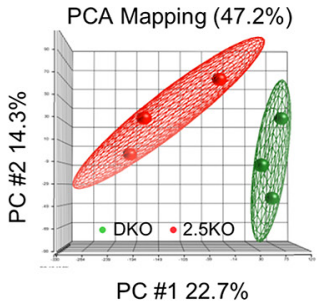

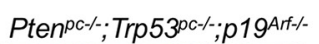
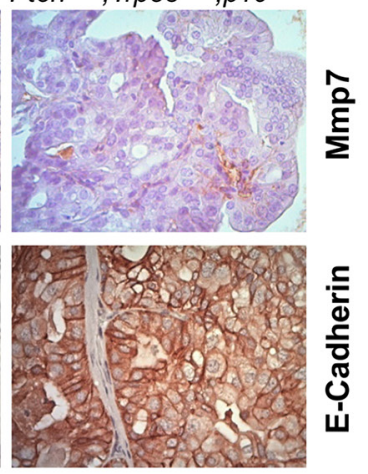

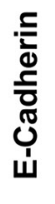
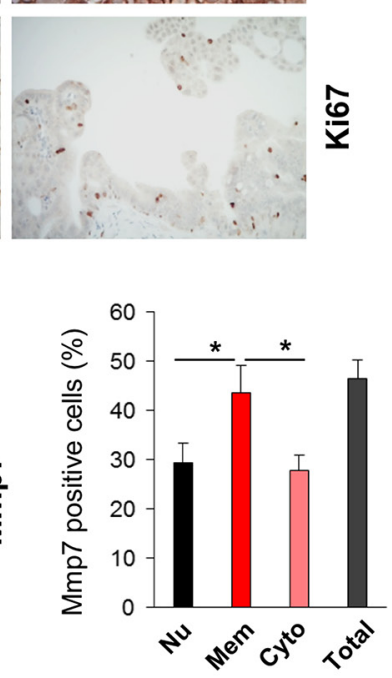

Figure 1: Mmp7 is elevated in prostate cancer of Pten/Trp53 mice but decreased by loss of p19 ${ }^{\text {Arf }}$ in vivo. (A) Gene heat

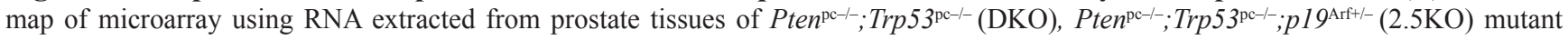
mice at 6 months of age. Right panel: data distribution of samples in indicated groups. Please refer Supplementary Tables S1 and S2 for the list of selected genes with fold changes. (B) Levels of Mmp7, E-Cadherin and Ki67 in anterior prostates of Wt, Pten ${ }^{\mathrm{pc}-/-}$; Trp53 ${ }^{\mathrm{pc}-/-}$, and Pten $^{\mathrm{pc}-/-} ; \operatorname{Trp} 53^{\mathrm{pc}-/-} ;$ p $19^{\mathrm{Arf}-/}$ mutant mice at 6 months of age. (C) Mmp7 in nucleus of malignant cells in prostate tumors of Pten ${ }^{\mathrm{pc}-/-}$;rp $53^{\mathrm{pc}-/-}$ mice. Mmp7 expression in nucleus ( $\mathrm{Nu}$ ) (arrows in black color), and Mmp7 at membrane and cytoplasm (Mem/Cyto) (arrows in red color). Right panel: Quantification analysis of cellular localization of Mmp7 expression. 


\section{MMP7 binds ARF in nucleus to promote cell migration}

The results above encouraged us to investigate whether MMP7 interacts with ARF to potentiate the oncogenic function for $\mathrm{PCa}$ progression. To examine the MMP7-ARF binding, we overexpressed GFP-MMP7 and Myc-p14 ${ }^{\mathrm{ARF}}$ in HEK293T cells and then performed coimmunoprecipitations (co-IP) with GFP or Myc antibody. Co-IP results demonstrated that MMP7 indeed binds ARF physically, while IgG control samples do not show any binding signals (Figure 4A). As MMP7 is predominantly localized at membrane and cytoplasmic compartments of cells such as Golgi [24], the aberrant interactions of MMP7 and ARF in nucleus propelled us to understand the promotion on $\mathrm{PCa}$ progression. We overexpressed MMP7 and p14 ${ }^{\mathrm{ARF}}$ in 22Rv1 PCa cells that have the undetectable levels of MMP7 and ARF. As predicted,
MMP7 protein was indeed co-localized with p14 ARF in the nucleus of 22Rv1 cells, while MMP7 alone was exclusively limited to cytosol and membrane (Figure 4B). These data further supported that $\mathrm{p} 14^{\mathrm{ARF}}$ is required for nuclear MMP7, in agreement with the results from $\mathrm{p} 14^{\mathrm{ARF}}$ knockdown (Figure 2C and 2D). We investigated the synergistic effects of MMP7 and ARF overexpression on cell migration. As shown, the combined overexpression of MMP7 and $\mathrm{p} 14^{\mathrm{ARF}}$ significantly increased cell migration of 22Rv1 cells as compared to the control (Figure 4C). Moreover, MMP7 knockdown decreased the wound healing of PC3 cells (Supplementary Figure S5). As ARF is a marker of nucleolus $[25,26]$, these lines of evidence implicate that MMP7 binding to ARF in nucleolus may contribute to rRNA biogenesis and protein synthesis in PCa cells. Our results indicate that ARF potentiates the oncogenic functions of MMP7 in nucleus for $\mathrm{PCa}$ progression.

\section{A}
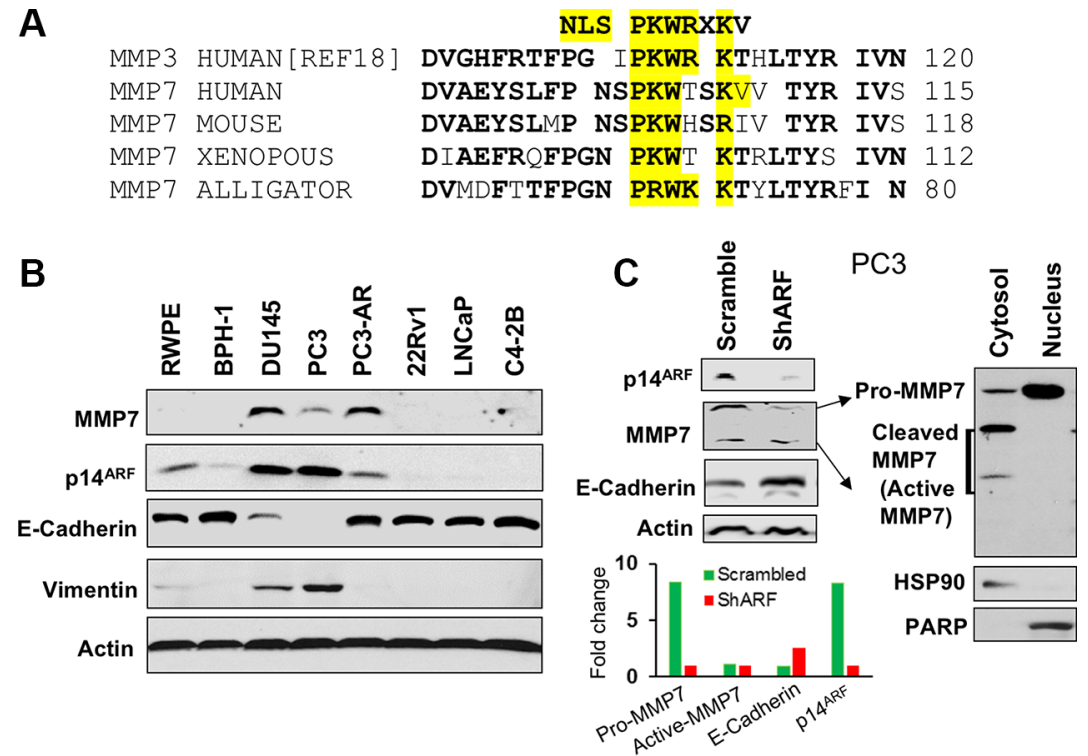

\section{D}
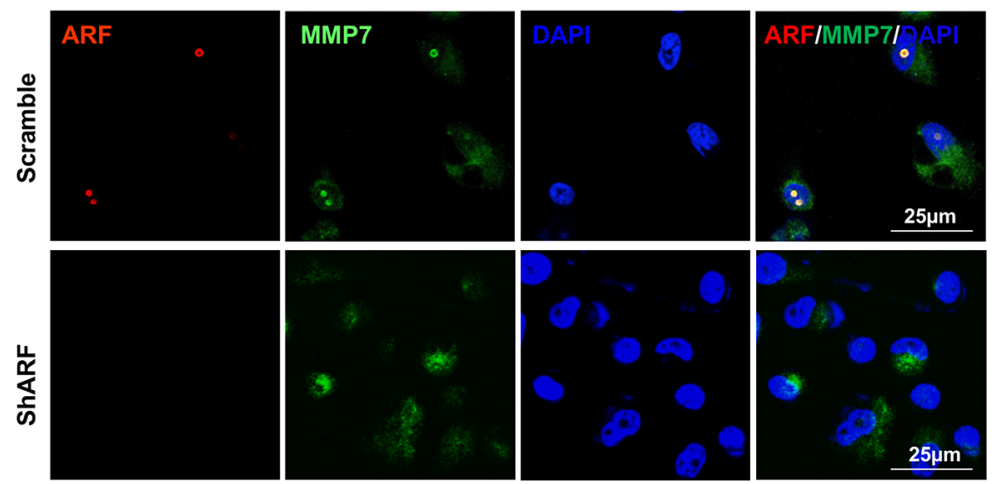

Figure 2: The expression and nuclear localization of MMP7 are decreased by p14 $^{\mathrm{ARF}}$ knockdown in human prostate cancer cells. (A) The nucleus localization signal (NLS) is conserved in MMP family among species. (B) MMP7 and p14 ${ }^{\mathrm{ARF}}$ are concomitantly elevated in prostate cancer cells, and MMP7 levels are inversely correlated with E-Cadherin in prostate cancer cells. (C) Western blot analysis demonstrates ARF knockdown results in a reduction of MMP7 levels with an increase of E-Cadherin in PC3 cells. MMP7 in nucleus is the pro-form not the active form of MMP7 protein. (D) Immunofluorescence (IF) images show the decreased MMP7 in nucleus of PC3 cells upon ARF knockdown. 


\section{MMP7 correlates with ARF expression levels in human prostate cancer specimens}

Given the decisive role of ARF and MMP7 interactions on tumor progression in vitro and in vivo, we examined the correlation between MMP7 and ARF in human PCa specimens. We performed IHC staining of MMP7 and ARF in PCa tissue microarrays (TMA) containing various stages and Gleason scores of primary cancer specimens. Notably, MMP7 was detected primarily in malignant cells of cancer lesions, with the intensive staining at the cytoplasm and plasma membrane (Figure 5A, arrows indicated). Most importantly, elevated MMP7 was accumulated in the nucleus of malignant cells (Figure 5A).
MMP7 was detected barely in luminal epithelial cells of normal tissues, although the weak staining was observed at the cytoplasm and plasma membrane of basal cells. Similar to ARF, MMP7 levels were significantly elevated in advanced stages (IV) and high Gleason scores of PCa as compared to that in stages II PCa with low Gleason scores and normal tissues (Figure 5B). Similar to MMP7, ARF elevation was found in both epithelial and stroma cells of PCa specimens (Figure 5C). MMP7 levels were significantly correlated with ARF in $\mathrm{PCa}$ samples (Figure 5D, $\left.r_{\mathrm{s}}=0.997, P<0.001\right)$. Thus, our data demonstrated that ARF may increase MMP7 levels to boost tumor microenvironments in human advanced PCa. In summary, our results indicate that ARF may promote cell motility and
A
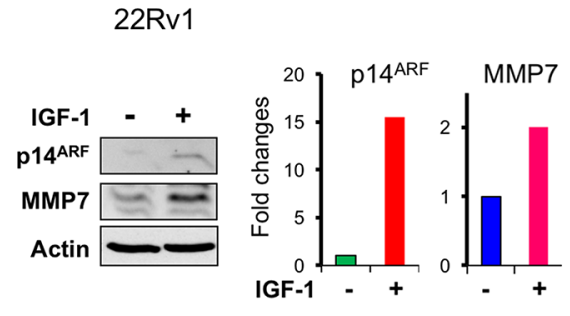

B

B TetO-p14ARF

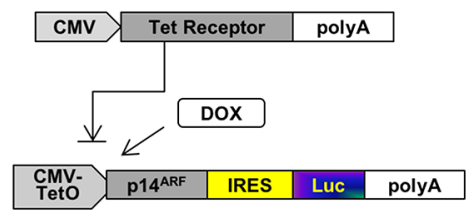

D

C4-2B-TetO-p14ARF
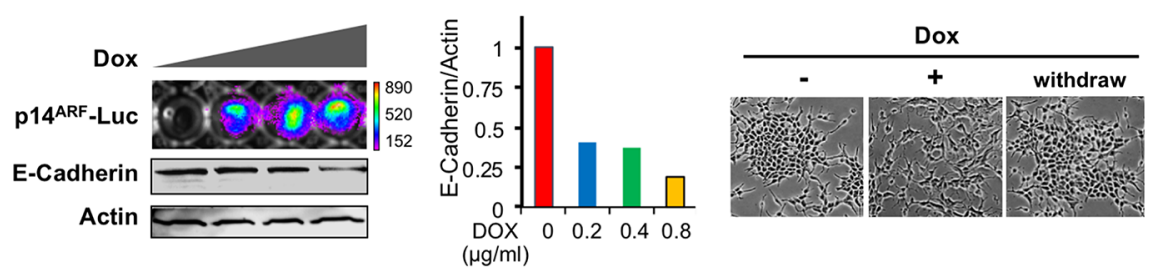

E

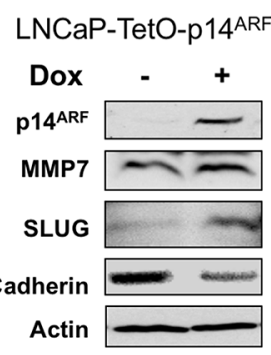

$\mathbf{F}$
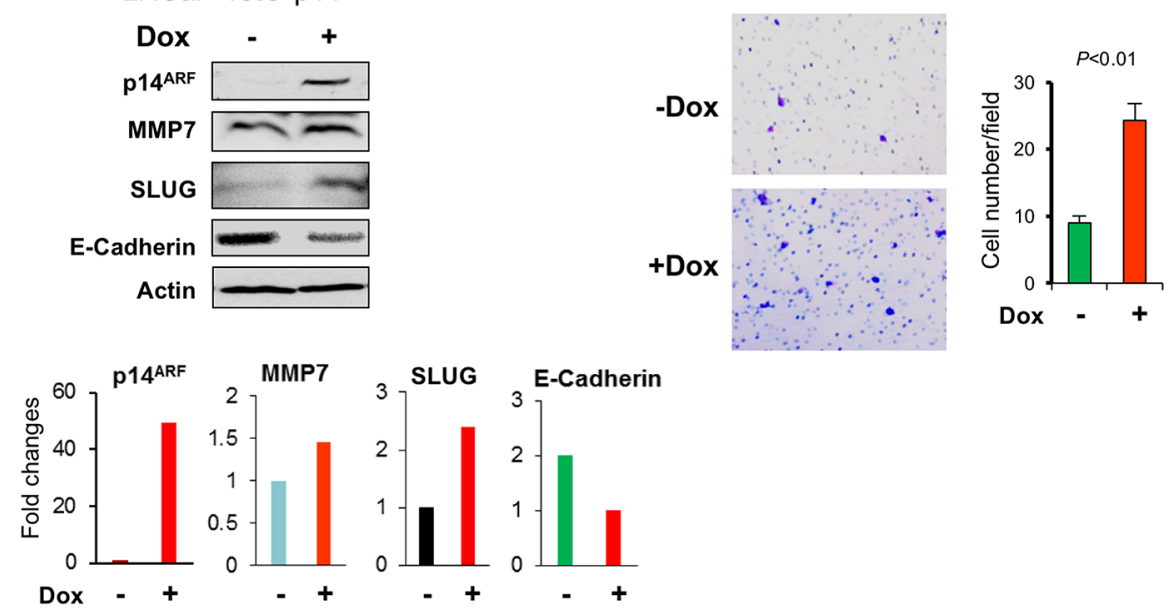

Figure 3: p14 ${ }^{\mathrm{ARF}}$ overexpression increases MMP7 levels with a decrease of E-Cadherin in human prostate cancer cells. (A) A concomitant induction of endogenous p14 ${ }^{\mathrm{ARF}}$ and MMP7 levels by IGF1 $(100 \mu \mathrm{g} / \mathrm{ml}, 6 \mathrm{hrs})$ in 22Rv1 PCa cells. (B) A schematic diagram of the doxycycline-inducible p14 ${ }^{\mathrm{ARF}}$ construct. (C) $\mathrm{p} 14^{\mathrm{ARF}}$ induction results in a decrease of E-Cadherin in C4-2B cells in a dose-dependent manner. Stable overexpression of p14 ${ }^{\mathrm{ARF}}$ fused with luciferase reporter gene was induced by doxycycline (Dox) treatment at $2 \mu \mathrm{g} / \mathrm{ml}$ for 2 days. Left panel: Luciferase intensity represented the expression level of $\mathrm{p} 14^{\mathrm{ARF}}$, and E-Cadherin was detected by Western blot. Right panel: quantification of E-Cadherin level. (D) The transition of cell morphology in C4-2B cells upon p14 ${ }^{\mathrm{ARF}}$ induction. (E) Changes of MMP7, SLUG, and E-Cadherin in LNCaP cells by p14ARF induction. (F) Increased cell migration of LNCaP cells upon p14 ${ }^{\mathrm{ARF}}$ induction. Migrated cells were counted from three fields and presented as mean values \pm s.d. $* * P<0.01$ indicates the statistical significance by Student's $t$-test $(n=3)$. 
cancer progression through upregulation of MMP7 upon PTEN/TP53 inactivation.

\section{DISCUSSION}

In this study, we elucidated in vitro and in vivo a novel mechanism in which ARF-MMP7 network contributes to PCa through enhancement of tumor microenvironments, at least in context of PTEN/TP53 loss. Moreover, the concomitant elevation of MMP7 and ARF in nucleus is associated with the malignancy of cancer cells. The nuclear localizations of MMPs have been reported in cancers, but

A 293T cells

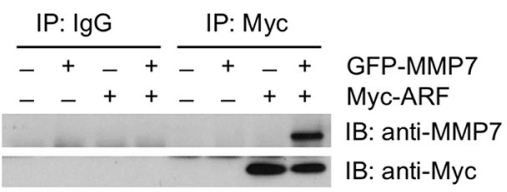

the molecular mechanism underlying this phenomenon remains elusive $[27,28]$. We demonstrated that ARF tumor suppressor stimulates the nuclear shuttling of MMP7 in PCa cells during cancer evolution. Our results provide some mechanistic explanations on the pro-proliferative and oncogenic-promoting roles of ARF in cancer cells [9, 15]. Collectively, the non-canonical ARF signaling may be more complicate than thought as it may be involved in ECM and EMT network in cancers.

$\mathrm{PCa}$ diseases result from the reprogramming of normal prostatic cells into invasive adenocarcinoma with metastasis potentials upon oncogenic insults and genetic
B
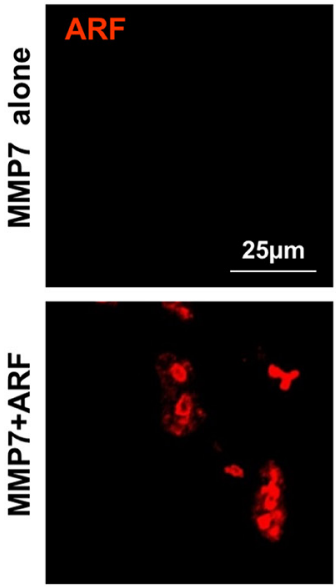

C

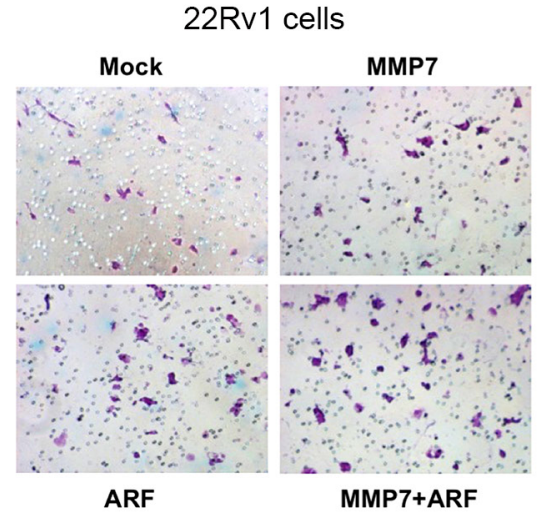

22Rv1 cells
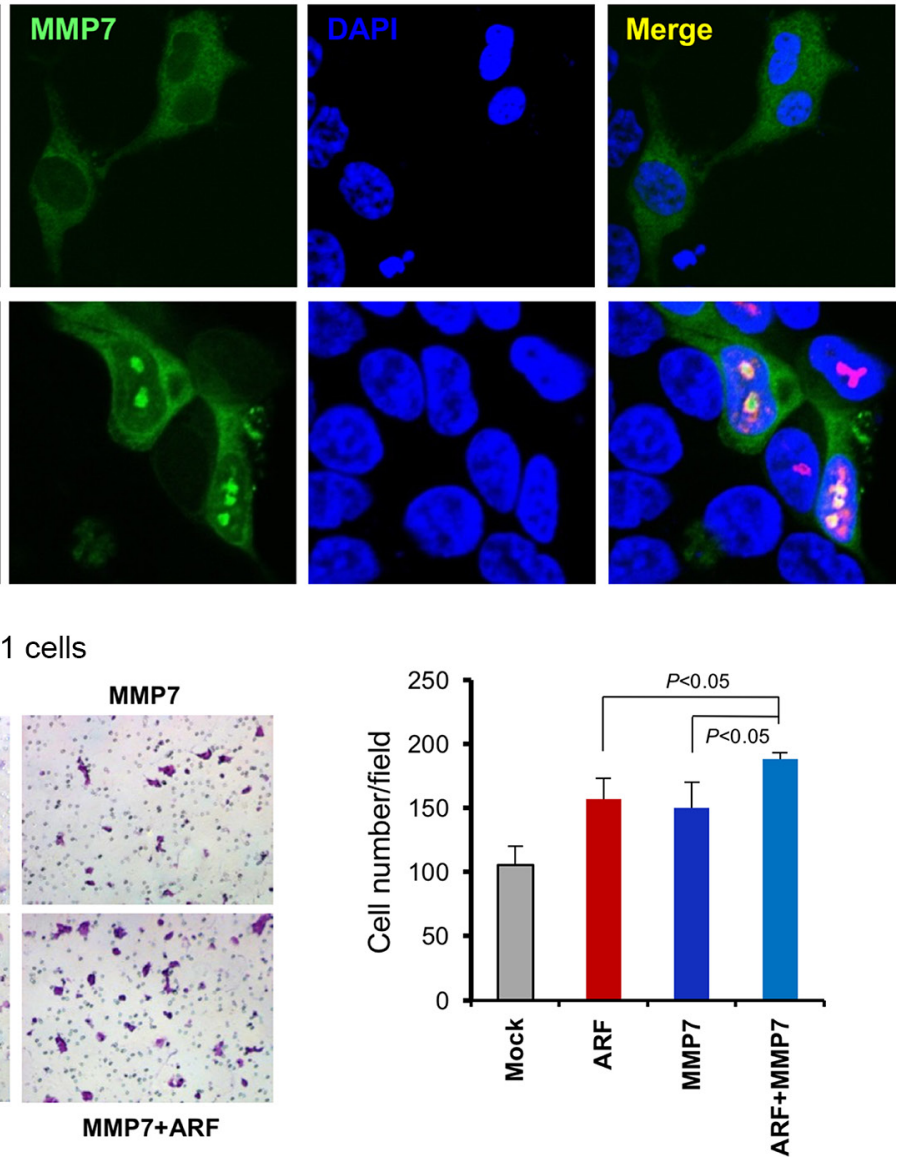

Figure 4: MMP7 interacts with ARF in nucleus to promote cell migration of human prostate cancer cells. (A) Coimmunoprecipitation of ARF and MMP7 in 293T cells. HEK293T cells were co-transfected with GFP-MMP7 and/or Myc-p14ARF plasmids. Cell lysates were immunoprecipitated with anti-GFP or anti-C-Myc followed with immunoblotting (IB) analysis with indicated antibodies. (B) ARF is required for the nuclear localization of MMP7 in 22Rv1 cells. 22Rv1 cells were transfected with GFP-MMP7, Myc-p14 ${ }^{\mathrm{ARF}}$ or GFP-MMP7 plus Myc-p14 ${ }^{\text {ARF }}$ plasmids using Lipofectamine 2000 (Invitrogen). Immunofluorescence (IF) images were collected as described previously [16]. (C) MMP7 cooperates with ARF to promote cell migration in 22Rv1 cells. 22Rv1 cells transfected with plasmids in (B) were subject to migration analysis. Left panel: the images on migrated cells on the membrane. Right panel: quantification of migrated cells promoted by MMP7 and ARF. 
alterations. MMP7 is associated with cell proliferation, mobility and cancer progression [19], yet the mechanism on MMP7 elevation in cancers is poorly understood. On the other hand, dysregulation of PTEN/PI3K pathways contributes to cancer progression through activation of downstream oncogenic pathways including MMP7, yet the mechanism on the aberrant elevation of MMP7 is puzzling [29]. Here we disclosure that MMP7 elevation induced by ARF potentiates EMT and tumor microenvironments for PCa progression using Pten/Trp53 mouse models and human PCa cells. Since ARF elevation by PTEN loss activates EMT [16], our findings provide a logic link with more evidence on the oncogenic network of PTEN/PI3K/ mTOR and ARF-MMP7-EMT pathways. This notion highlights a unique signaling pathway of PTEN, ARF and MMP7 and their oncogenic relevance in PCa (Figure 5E).

One intriguing phenomenon in this study is that ARF serves as a protein-binding docking of MMP7 in nucleus of PCa cells, and knockdown or inactivation of ARF abrogates nuclear MMP7 (Figures 2 and 4, and Supplementary

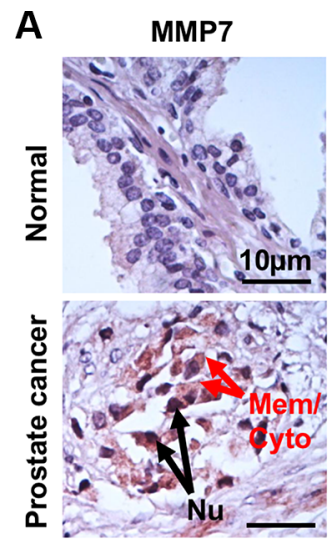

$\mathbf{C}$

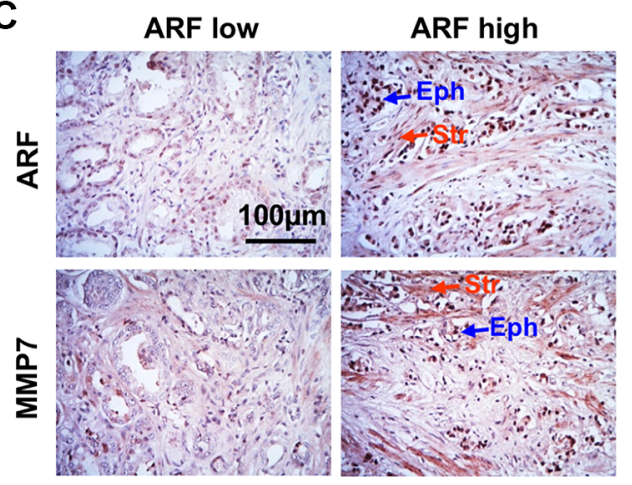

B Cancer Stage

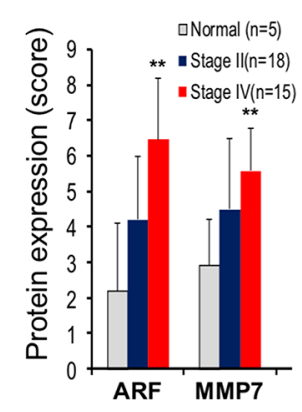

D

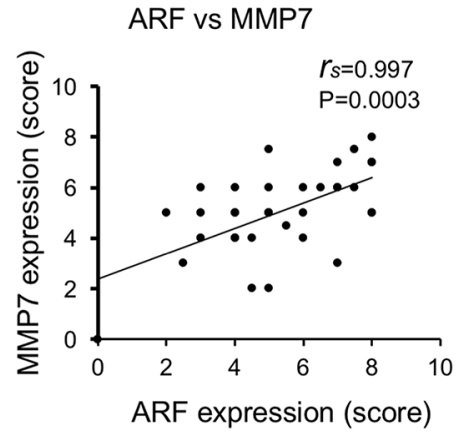

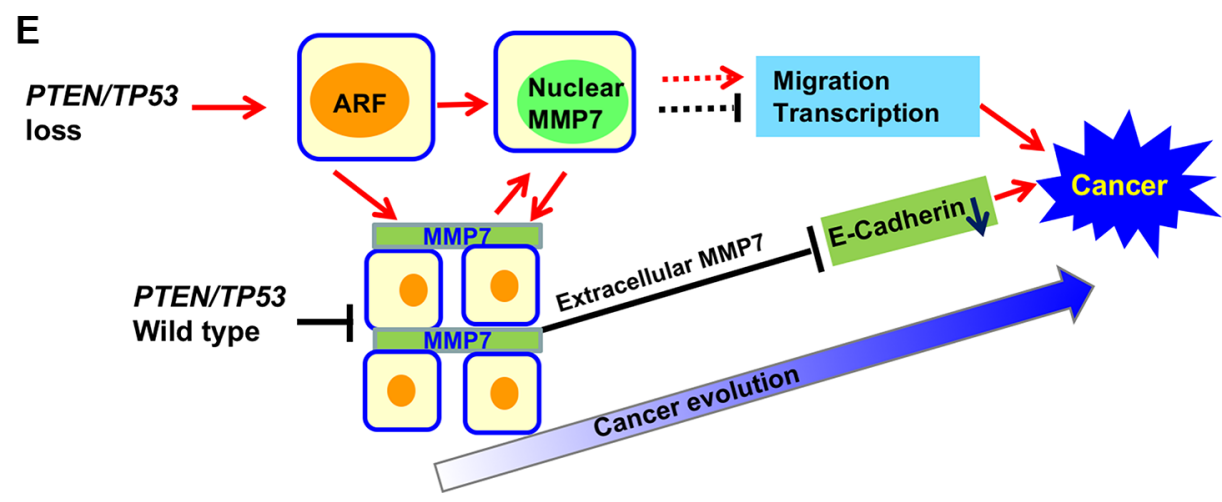

Figure 5: MMP7 correlates with ARF in human prostate cancer specimens. (A) IHC staining reveals that MMP7 levels are increased in prostate cancer specimens as compared to normal tissues. Arrows indicate MMP7 expression on the membrane (Mem), the cytoplasm (Cyto) or the nucleus (Nu) of prostate cancer samples. (B) Levels of ARF and MMP7 expression among normal, stages II and IV of prostate cancer. Student's $t$-test was used to compare the expression levels at different stages. Kruskal-Wallis $H$ test was used to determine the correlations between Gleason scores and expression levels of ARF/MMP7. (C) IHC staining of ARF and MMP7 in epithelial and stromal cells of human prostate cancer specimens. Eph: epithelial cells, Str: stromal cells. (D) A significant correlation between ARF and MMP7 expression in prostate cancer specimens. Spearman's coefficient method was applied to determine the correlation. (E) Signaling networks of PTEN/TP53, ARF, MMP7, and E-Cadherin for prostate cancer progression and evolution. 
Figure S2). Increasing evidence showed that, in addition to ECM, MMPs are also found in nucleus of cells, underscoring the pivotal roles of nuclear MMPs on cellular functions [18, 27, 30]. For example, nuclear MMP2 cleaves PARP in nucleus for the degradation to promote cell invasion [27, 31]. Nuclear MMP3 activates CCN2 gene transcription for IL-6-mediated cell migration [28, 32]. As MMP7 is associated with the malignant features of cancers $[33,34]$, our findings offer novel and valuable insights into understanding the functions of MMP7 in PCa as following: 1) MMP7 shuttles to nucleus without being cleaved, and it certainly deserves further investigation to elucidate the mechanisms on shuttling and the biological actions of MMP7 in nucleus of PCa cells; 2) ARF binds pro-MMP7 $(\sim 30 \mathrm{kDa})$ not active-MMP7 $(\sim 19 \mathrm{kDa})$ in nucleus, and it will be interesting to know these binding domains of MMP7 and ARF proteins; 3) Nuclear factors associated with ARFMMP7 in nuclear compartments promote cell migration of PCa cells. ARF knockdown decreases MMP7 at both the transcription and the protein stability (Supplementary Figures S3 and S4), suggesting the ARF-MMP7 binding in nucleus may prevent pro-MMP7 from degradation. In addition to the NLS partially conserved in other MMPs [18] (data not shown), MMP7 protein contains a SUMOylation site at K64 as predicted by SUMOplot ${ }^{\mathrm{TM}}$ (www.abgent. com/sumoplot). As ARF determines SLUG stability in nucleus through SUMOylation [16], ARF may couple with several nuclear proteins to promote the SUMOylationmediated stability of MMP7 in nucleus. Therefore, it is likely that MMP7 imports and accumulates in nucleus via a mechanism independent of NLS for stabilization after ARFmediated SUMOylation.

In summary, our findings highlight a novel mechanism of ARF-MMP7 signaling in which ARF elevation promotes cell invasion through increasing oncogenic potentials of MMP7 for E-Cadherin downregulation in PCa progression and evolution (Figure 5E). Given that MMP7 is a potential "druggable" target on cancers in clinics, mechanisms on ARF-MMP7 inhibition would produce valuable information on the development of effective therapeutic treatments. A combined targeting of ARF and MMP7 would be of great significance in treatment of advanced PCa.

\section{MATERIALS AND METHODS}

\section{Mutant mice and tumor analysis}

Mice were bred and maintained in accordance with IACUC guidelines at Meharry Medical College. Pten and Trp53 conditional knockout in prostates are controlled by Probasin-Cre recombination [35]. Generation, genotyping and prostate tumor analysis of wild type $(W t), \mathrm{Pten}^{\mathrm{pc}-/-}$; Trp53 $3^{\mathrm{pc}-1-}$ double-mutant (referred to as Pten/Trp53), and Pten $^{\mathrm{pc}-/-} ; \operatorname{Trp}^{\mathrm{pc}-/-} ; p 19^{\mathrm{Arf}+/}$ as well as Pten $^{\mathrm{pc}-/-} ; \operatorname{Trp}^{\mathrm{p}} 3^{\mathrm{pc}-/-}$; $p 19^{\text {Arf-/- }}$ triple-mutant mice (referred to as Pten/Trp53/ p19 $\left.{ }^{\text {Arf }}\right)$ were performed as described previously $[9,16]$.

\section{Transcriptome microarray analysis}

Total RNA was subject to gene expression profiling using the Affymetrix Mouse Gene 1.1-ST v1 whole transcriptome array (Affymetrix, Santa Clara, CA). Data analysis was performed at Meharry Bioinformatics Core (Nashville, TN) using Partek Genomics Suite version 6.6 (Partek Inc., St. Louis, MO). Affymetrix CEL files were normalized using the Robust Multi-array Average (RMA) algorithm [36, 37]. Fold changes of transcriptome levels and the significance analyses were calculated using ANOVA. Significantly changed transcripts were defined as having $\mathrm{a} \geq \pm 2.0$ fold expression from controls and an ANOVA $P$-value $\leq 0.05$.

\section{Tet-inducible cell lines, IGF treatment and cell migration assay}

Tet-inducible cell lines were established by inserting p14 ${ }^{\mathrm{ARF}}$-GFP-IRES-Luciferase cassettes into the Tet-on system (Clontech). First, the Tet receptor (Tet-R) expressing plasmids were introduced into $\mathrm{LNCaP}$ or $\mathrm{C} 4-2 \mathrm{~B}$ cell lines and selected by zeocin antibiotics. Secondly, stable cell lines of Tet-R-LNCaP or Tet-R-C4-2B were transfected with plasmids containing p14 ${ }^{\mathrm{ARF}}$-GFP-IRES-Luciferase and selected by Puromycin. For IGF stimulation, 22Rv1 cells were treated with IGF at a concentration of $100 \mu \mathrm{g} /$ $\mathrm{ml}$ for 6 hrs. For migration assay, cells were grown until $70-80 \%$ confluence followed by serum starvation for $40 \mathrm{hrs}$. Cells were seeded at a density of $5 \times 10^{4} /$ well for PC3 cells or $7.5 \times 10^{4} /$ well for LNCaP cells in serum-free medium into the upper chamber with $8 \mu \mathrm{m}$ polyethylene terephalate membrane filters (Falcon cell culture insert, Becton-Dickinson). Cells were allowed to migrate for $24 \mathrm{hrs}$ for PC3 cells or $48 \mathrm{hrs}$ for LNCaP cells in a humidified chamber at $37^{\circ} \mathrm{C}$ with $5 \% \mathrm{CO}_{2}$. Non-migrant cells on the upper side of the filters were removed using cotton swab. Filters containing cells were fixed with $4 \%$ formaldehyde for $15 \mathrm{~min}$, and cells in the lower filter were stained with $0.5 \%$ crystal violet and counted from three microscopy fields.

\section{Western blotting and fractionation analysis}

For Western blotting, cell lysates were prepared in RIPA buffer $[1 \times$ PBS, $1 \%$ Nonidet P-40, $0.5 \%$ sodium deoxycholate, $0.1 \%$ SDS, and protease inhibitor cocktail (Roche)], and then were subject to standard procedures of SDS-PAGE and antibody detection [26]. Antibodies used were: $\beta$-actin (AC-74, 1:5000, Sigma), MMP7 (monoclonal, JL07, sc80825, 1:500, Santa Cruz), p14 ${ }^{\text {ARF }}$ (14P02, 1:750, NeoMarkers), E-Cadherin (sc33743, 1:500, Santa Cruz). Cell fractionation was performed using NE-PER nuclear and cytoplasmic protein extraction kit or subcellular protein fractionation kit (Thermo Scientific) followed by standard procedures of SDS-PAGE and antibody detection for IB using primary antibodies to: MMP7 (monoclonal, JL07, sc80825, 1:500, Santa Cruz or ab176325, 1:1000, Abcam), 
PARP (9542, 1:1000, Cell Signaling), HSP90 (H114, sc-7947, 1:1000, Santa Cruz).

\section{Co-immunoprecipitation (Co-IP), immunofluorescence (IF) and ARF knockdown by ShRNA}

For Co-IP experiment, HEK293T cells were transfected with GFP-tagged MMP7 and Myc-tagged p14 ${ }^{\mathrm{ARF}}$ plasmids. Cell lysates were collected $48 \mathrm{hrs}$ posttransfection in RIPA buffer (1XPBS, pH 7.4, $2 \mathrm{mM}$ EDTA, 1\% Triton X-100, $0.5 \%$ sodium deoxycholate, and protease inhibitor), and used for immunoprecipitation by incubating with anti-Myc or anti-GFP antibodies for $1 \mathrm{hr}$ in cold room [26]. The $\mathrm{A} / \mathrm{G}$ plus agarose beads were used for immunoprecipitation of antibody-antigen complex, and IgG was used as a control. The reactions were stopped by adding SDS loading buffer, and samples were subject to Western blotting. For IF experiment, cells were grown on coverslips for 2 days and fixed for $15 \mathrm{~min}$ in ice-cold methanol. Cells on slides were probed with following antibodies: p14 ${ }^{\mathrm{ARF}}$ (14P02, 1:200, NeoMarkers), MMP7 (monoclonal, JL07, sc80825, 1:50, Santa Cruz) or MMP7 (polyclonal, AF907, 1:200, R \& D system) overnight at $4^{\circ} \mathrm{C}$, followed by incubation with Alexa Fluor 568 or 488 dye conjugated to anti-mouse or rabbit IgG (Invitrogen) antibodies. Slides were washed and mounted with Vectashield medium (Vector Laboratories), and images were scanned with confocal microscopy [38]. p14 ${ }^{\mathrm{ARF}}$ knockdown in PC3 cells were generated using shRNA as described previously $[16,26]$.

\section{Statistical analysis}

Data were evaluated using two-tail student's $t$-test, the values of $P \leq 0.05$ were considered statistically significant. In particular, to determine the significance of differences in human prostate specimens, data were evaluated using Kruskal-Wallis H test or Spearman's test as indicated in figure legends.

\section{Immunohistochemistry (IHC) and prostate tissue microarrays (TMA)}

For IHC analysis, after mouse tissues were fixed and processed, sections were probed using primary antibodies: E-Cadherin (24E10, 1:400, Cell Signaling), MMP7 antibody (D4H5-XP, 3801, 1:50, Cell Signaling), or Ki-67 antibody (NCL-Ki-67p, 1:200, Novocastra). Human prostate tissue microarrays (TMA) containing $\mathrm{PCa}(n=35)$ and normal tissues $(n=5)$ in duplicates (US Biomax) were used for IHC staining with antibodies of p14 ${ }^{\mathrm{ARF}}$ (4C6/4, 1:50, 2407, Cell Signaling) and MMP7 (JL07, sc80825, 1:50, Santa Cruz). The staining intensities were scored as previously described [39], and analyzed for the correlation between ARF and MMP7.

\section{Abbreviations}

PCa-prostate cancer, ECM-extracellular matrix, EMT-epithelial-mesenchymal transition, MMP-matrix metalloproteinase, IHC-immunohistochemistry.

\section{ACKNOWLEDGMENTS AND FUNDING}

We would like to thank Dr. Pier Paolo Pandolfi for his generous support with mouse models, Drs. LaMonica V. Stewart, Simon W. Hayward and Robert J. Matusik for providing prostate cancer cell lines. Y.X. and Z.C. conceived and designed the experiments, and wrote the manuscript. Y.X., W.L., S.L., Q.Y., J.S.G., S.A.S., and S.P. performed the experiments and data analysis. This work was supported in part by Social Policy grant 2.23 to Y.X. and NIH grants MD004038, CA091408, CA163069 and UL1 TR000445-06. Meharry Medical College Morphology Core is supported in part by NIH grants U54 MD007593, G12 MD007586, R24 DA036420, and S10RR0254970.

\section{CONFLICTS OF INTEREST}

The authors declare that they have no conflicts of interests.

\section{REFERENCES}

1. Torre LA, Bray F, Siegel RL, Ferlay J, Lortet-Tieulent J, Jemal A. Global cancer statistics, 2012. CA Cancer J Clin. 2015; 65:87-108.

2. Zhang L, Yang BX, Zhang HT, Wang JG, Wang HL, Zhao XJ. Prostate cancer: an emerging threat to the health of aging men in Asia. Asian J Androl. 2011; 13:574-578.

3. Thomas G, Jacobs KB, Yeager M, Kraft P, Wacholder S, Orr N, Yu K, Chatterjee N, Welch R, Hutchinson A, Crenshaw A, Cancel-Tassin G, et al. Multiple loci identified in a genome-wide association study of prostate cancer. Nat Genet. 2008; 40:310-315.

4. Kote-Jarai Z, Olama AA, Giles GG, Severi G, Schleutker J, Weischer M, Campa D, Riboli E, Key T, Gronberg H, Hunter DJ, Kraft P, et al. Seven prostate cancer susceptibility loci identified by a multi-stage genome-wide association study. Nat Genet. 2011; 43:785-791.

5. Berger MF, Lawrence MS, Demichelis F, Drier Y, Cibulskis K, Sivachenko AY, Sboner A, Esgueva R, Pflueger D, Sougnez C, Onofrio R, Carter SL, et al. The genomic complexity of primary human prostate cancer. Nature. 2011; 470:214-220.

6. Hutchinson L. Genetics: predictor for prostate cancer. Nat Rev Clin Oncol. 2011; 8:193.

7. Pourmand G, Ziaee AA, Abedi AR, Mehrsai A, Alavi HA, Ahmadi A, Saadati HR. Role of PTEN gene in progression of prostate cancer. Urol J. 2007; 4:95-100. 
8. Parsons R, Simpson L. PTEN and cancer. Methods Mol Biol. 2003; 222:147-166.

9. Chen Z, Carracedo A, Lin HK, Koutcher JA, Behrendt N, Egia A, Alimonti A, Carver BS, Gerald W, Teruya-Feldstein J, Loda M, Pandolfi PP. Differential p53-independent outcomes of p19(Arf) loss in oncogenesis. Sci Signal. 2009; 2:ra44.

10. Ha L, Ichikawa T, Anver M, Dickins R, Lowe S, Sharpless NE, Krimpenfort P, Depinho RA, Bennett DC, Sviderskaya EV, Merlino G. ARF functions as a melanoma tumor suppressor by inducing p53-independent senescence. Proc Natl Acad Sci U S A. 2007; 104:10968-10973.

11. Chen D, Shan J, Zhu WG, Qin J, Gu W. Transcriptionindependent ARF regulation in oncogenic stress-mediated p53 responses. Nature. 2010; 464:624-627.

12. Gallagher SJ, Kefford RF, Rizos H. The ARF tumour suppressor. Int J Biochem Cell Biol. 2006; 38: 1637-1641.

13. Kim WY, Sharpless NE. The regulation of INK4/ARF in cancer and aging. Cell. 2006; 127:265-275.

14. Sherr CJ. Divorcing ARF and p53: an unsettled case. Nat Rev Cancer. 2006; 6:663-673.

15. Humbey O, Pimkina J, Zilfou JT, Jarnik M, DominguezBrauer C, Burgess DJ, Eischen CM, Murphy ME. The ARF tumor suppressor can promote the progression of some tumors. Cancer Res. 2008; 68:9608-9613.

16. Xie Y, Liu S, Lu W, Yang Q, Williams KD, Binhazim AA, Carver BS, Matusik RJ, Chen Z. Slug regulates E-cadherin repression via p19Arf in prostate tumorigenesis. Mol Oncol. 2014; 8:1355-1364.

17. Mehner C, Hockla A, Miller E, Ran S, Radisky DC, Radisky ES. Tumor cell-produced matrix metalloproteinase 9 (MMP-9) drives malignant progression and metastasis of basal-like triple negative breast cancer. Oncotarget. 2014; 5:2736-2749. doi: 10.18632/oncotarget.1932.

18. Si-Tayeb K, Monvoisin A, Mazzocco C, Lepreux S, Decossas M, Cubel G, Taras D, Blanc JF, Robinson DR, Rosenbaum J. Matrix metalloproteinase 3 is present in the cell nucleus and is involved in apoptosis. Am J Pathol. 2006; 169:1390-1401.

19. Fukuda A, Wang SC, Morris JPt, Folias AE, Liou A, Kim GE, Akira S, Boucher KM, Firpo MA, Mulvihill SJ, Hebrok M. Stat3 and MMP7 contribute to pancreatic ductal adenocarcinoma initiation and progression. Cancer Cell. 2011; 19:441-455.

20. Schummer M, Drescher C, Forrest R, Gough S, Thorpe J, Hellstrom I, Hellstrom KE, Urban N. Evaluation of ovarian cancer remission markers HE4, MMP7 and Mesothelin by comparison to the established marker CA125. Gynecol Oncol. 2012; 125:65-69.

21. Ip YC, Cheung ST, Fan ST. Atypical localization of membrane type 1-matrix metalloproteinase in the nucleus is associated with aggressive features of hepatocellular carcinoma. Mol Carcinog. 2007; 46:225-230.

22. Kosugi S, Hasebe M, Matsumura N, Takashima $\mathrm{H}$, Miyamoto-Sato E, Tomita M, Yanagawa H. Six classes of nuclear localization signals specific to different binding grooves of importin alpha. J Biol Chem. 2009; 284:478-485.

23. Kalderon D, Roberts BL, Richardson WD, Smith AE. A short amino acid sequence able to specify nuclear location. Cell. 1984; 39:499-509.

24. Gorodeski GI. Estrogen decrease in tight junctional resistance involves matrix-metalloproteinase-7-mediated remodeling of occludin. Endocrinology. 2007; 148:218-231.

25. Korgaonkar C, Hagen J, Tompkins V, Frazier AA, Allamargot C, Quelle FW, Quelle DE. Nucleophosmin (B23) targets ARF to nucleoli and inhibits its function. Mol Cell Biol. 2005; 25:1258-1271.

26. Lu W, Xie Y, Ma Y, Matusik RJ, Chen Z. ARF represses androgen receptor transactivation in prostate cancer. Mol Endocrinol. 2013; 27:635-648.

27. Kwan JA, Schulze CJ, Wang W, Leon H, Sariahmetoglu M, Sung M, Sawicka J, Sims DE, Sawicki G, Schulz R. Matrix metalloproteinase-2 (MMP-2) is present in the nucleus of cardiac myocytes and is capable of cleaving poly (ADPribose) polymerase (PARP) in vitro. FASEB J. 2004; 18 : 690-692.

28. Eguchi T, Kubota S, Kawata K, Mukudai Y, Uehara J, Ohgawara T, Ibaragi S, Sasaki A, Kuboki T, Takigawa M. Novel transcription-factor-like function of human matrix metalloproteinase 3 regulating the CTGF/CCN2 gene. Mol Cell Biol. 2008; 28:2391-2413.

29. Zhang Q, Liu S, Ge D, Zhang Q, Xue Y, Xiong Z, AbdelMageed AB, Myers L, Hill SM, Rowan BG, Sartor O, Melamed J, et al. Interleukin-17 promotes formation and growth of prostate adenocarcinoma in mouse models. Cancer Res. 2012; 72:2589-2599.

30. Mannello F, Medda V. Nuclear localization of matrix metalloproteinases. Prog Histochem Cytochem. 2012; 47:27-58.

31. Voll EA, Ogden IM, Pavese JM, Huang X, Xu L, Jovanovic BD, Bergan RC. Heat shock protein 27 regulates human prostate cancer cell motility and metastatic progression. Oncotarget. 2014; 5:2648-2663. doi: 10.18632/oncotarget.1917.

32. Jiang YN, Yan HQ, Huang XB, Wang YN, Li Q, Gao FG. Interleukin 6 trigged ataxia-telangiectasia mutated activation facilitates lung cancer metastasis via MMP-3/MMP-13 up-regulation. Oncotarget. 2015; 6:40719-40733. doi: 10.18632/oncotarget.5825.

33. Sizemore ST, Sizemore GM, Booth CN, Thompson CL, Silverman P, Bebek G, Abdul-Karim FW, Avril S, Keri RA. Hypomethylation of the MMP7 promoter and increased expression of MMP7 distinguishes the basal-like breast cancer subtype from other triple-negative tumors. Breast Cancer Res Treat. 2014; 146:25-40.

34. Grindel BJ, Martinez JR, Pennington CL, Muldoon M, Stave J, Chung LW, Farach-Carson MC. Matrilysin/matrix metalloproteinase-7(MMP7) cleavage of perlecan/HSPG2 creates a molecular switch to alter prostate cancer cell behavior. Matrix Biol. 2014; 36:64-76. 
35. Chen Z, Trotman LC, Shaffer D, Lin HK, Dotan ZA, Niki M, Koutcher JA, Scher HI, Ludwig T, Gerald W, CordonCardo C, Pandolfi PP. Crucial role of p53-dependent cellular senescence in suppression of Pten-deficient tumorigenesis. Nature. 2005; 436:725-730.

36. Irizarry RA, Bolstad BM, Collin F, Cope LM, Hobbs B, Speed TP. Summaries of Affymetrix GeneChip probe level data. Nucleic Acids Res. 2003; 31:e15.

37. Irizarry RA, Hobbs B, Collin F, Beazer-Barclay YD, Antonellis KJ, Scherf U, Speed TP. Exploration, normalization, and summaries of high density oligonucleotide array probe level data. Biostatistics. 2003; 4:249-264.
38. Lu W, Liu S, Li B, Xie Y, Adhiambo C, Yang Q, Ballard BR, Nakayama KI, Matusik RJ, Chen Z. SKP2 inactivation suppresses prostate tumorigenesis by mediating JARID1B ubiquitination. Oncotarget. 2015; 6:771-788. doi: 10.18632/ oncotarget.2718.

39. Ji H, Wang J, Nika H, Hawke D, Keezer S, Ge Q, Fang B, Fang X, Fang D, Litchfield DW, Aldape K, Lu Z. EGFinduced ERK activation promotes CK2-mediated disassociation of alpha-Catenin from beta-Catenin and transactivation of beta-Catenin. Mol Cell. 2009; 36:547-559. 